\title{
Approximate Homomorphisms and Derivations on Normed Lie Triple Systems
}

\author{
Hark-Mahn Kim and Juri Lee \\ Department of Mathematics, Chungnam National University, Daejeon 305-764, Republic of Korea \\ Correspondence should be addressed to Juri Lee; annans@nate.com
}

Received 17 December 2013; Revised 19 March 2014; Accepted 18 April 2014; Published 7 May 2014

Academic Editor: Janusz Brzdęk

Copyright ( $) 2014$ H.-M. Kim and J. Lee. This is an open access article distributed under the Creative Commons Attribution License, which permits unrestricted use, distribution, and reproduction in any medium, provided the original work is properly cited.

We investigate the generalized Hyers-Ulam stability of homomorphisms and derivations on normed Lie triple systems for the following generalized Cauchy-Jensen additive equation $r_{0} f\left(\left(s \sum_{j=1}^{p} x_{j}+t \sum_{j=1}^{d} y_{j}\right) / r_{0}\right)=s \sum_{j=1}^{p} f\left(x_{j}\right)+t \sum_{j=1}^{d} f\left(y_{j}\right)$, where $r_{0}, s$, and $t$ are nonzero real numbers. As a results, we generalize some stability results concerning this equation.

\section{Introduction}

The stability problem of functional equations originated from a question of Ulam [1], concerning the stability of group homomorphisms.

Let $(G, \circ)$ be a group and let $(H, \star, d)$ be a metric group with the metric $d(\cdot, \cdot)$. Given $\varepsilon>0$, there exist a scalar $\delta=$ $\delta(\varepsilon)>0$ such that if a mapping $f: G \rightarrow H$ satisfies the inequality $d(f(x \circ y), f(x) \star f(y))<\delta$ for all $x, y \in G$, then a homomorphism $F: G \rightarrow H$ exists with $d(f(x), F(x))<\varepsilon$ for all $x \in G$ ?

Hyers [2] gave the first affirmative partial answer to the question of Ulam for additive mappings on Banach spaces. Hyers' theorem was generalized by Aoki [3] for additive mappings and by Rassias [4] for linear mappings by considering an unbounded Cauchy difference. In 1990, Rassias [5] during 27th international symposium on functional equations asked the question whether such a theorem can also be proved for $p \geq 1$. In 1991, Gajda [6] following the same approach as in Rassias [5] gave an affirmative solution to this question for $p>1$. It was proved by Gajda [6], as well as by Rassias and Šemrl [7] that one cannot prove Rassias' type theorem when $p=1$.

During the last three decades, several stability problems of functional equations have been investigated by a number of mathematicians; compare or confer [8-12] and references therein.
Now, we introduce the concept of normed Lie triple systems. First of all, ternary algebraic operations were considered in 19th century by several mathematicians such as Cayley [13] who introduced the notion of cubic matrix which in turn was generalized by Kapranov et al. [14] in 1990. There are some applications, although they are still hypothetical, in the fractional quantum Hall effect, the nonstandard statistics, supersymmetric theory, and Yang-Baxter equation. The comments on physical applications of ternary structures can be found in [15-22].

A normed (Banach) Lie triple system is a normed (Banach) space $(A ;\|\cdot\|)$ with a trilinear mapping $(x, y, z) \mapsto$ $[x, y, z]$ from $A \times A \times A$ to $A$ satisfying the following axioms:

(i) $[x, y, z]=-[y, x, z]$,

(ii) $[x, y, z]+[y, z, x]+[z, x, y]=0$,

(iii) $[u, v,[x, y, z]]=[[u, v, x], y, z]+[x,[u, v, y], z]+$ $[x, y,[u, v, z]]$

(iv) $\|[x, y, z]\| \leq\|x\|\|y\|\|z\|$,

for all $u, v, x, y, z \in A$. The concept of Lie triple system was first introduced by Lister [23] (see also [24, 25]). Let $A$ and $B$ be normed Lie triple systems. A $\mathbb{C}$-linear mapping $H: A \rightarrow$ $B$ is said to be a Lie triple homomorphism if $H([x, y, z])=$ $[H(x), H(y), H(z)]$ for all $x, y, z \in A$. A $\mathbb{C}$-linear mapping $D: A \rightarrow A$ is called a Lie triple derivation if $D([x, y, z])=$ $[D(x), y, z]+[x, D(y), z]+[x, y, D(z)]$ for all $x, y, z \in A$. 
The third identity asserts that the mapping $D_{u, v}: x \mapsto$ $[u, v, x]$ is a (inner) derivation on $A[25]$.

Clearly, every Lie algebra is at the same time a Lie triple system via $[x, y, z]:=[[x, y], z]$, and our definition of a homomorphism (derivation) coincides with that of prehomomorphism (prederivation) on a Lie algebra. Also, if $U$ is an involutive automorphism of a Lie algebra $(L,[]$,$) ,$ then the eigenspace $E_{-1}(U)$ is a Lie triple system; see [25]. We remark that Lie triple systems are important since they give the structure of the tangent space of a symmetric space. Confer [25, 26] for reference. Also some applications of Lie triple systems can be found in Nambuòs approach by modifying the Heisenberg equation of motion [27].

Now, one of the interesting functional equations is the following general Cauchy-Jensen additive equation:

$$
r_{0} f\left(\frac{s \sum_{j=1}^{p} x_{j}+t \sum_{j=1}^{d} y_{j}}{r_{0}}\right)=s \sum_{j=1}^{p} f\left(x_{j}\right)+t \sum_{j=1}^{d} f\left(y_{j}\right)
$$

which is a generalization of Cauchy or Jensen additive equations, where $r_{0}, s$, and $t$ are nonzero real numbers and $f$ is a mapping between linear spaces. It is easy to see that a mapping $f$ satisfies the above Cauchy-Jensen additive equation if and only if $f(x)-f(0)$ is additive, where $f(0)=0$ if $r_{0} \neq s p+t d$. In this paper, we refine the generalized HyersUlam stability results of [25] for Lie triple homomorphisms and Lie triple derivations on Lie triple systems associated with the general Cauchy-Jensen additive equation (1) and then we apply our results to study stability theorems of Lie triple homomorphisms and Lie triple derivations associated with Cauchy-Jensen additive equation (1) on normed Lie triple systems, which can be regarded as ternary structures. The reader may be referred to [28-30] for some other related stability results on derivations.

Throughout this paper, suppose that $A$ is a normed Lie triple system with norm $\|\cdot\|_{A}$ and that $B$ is a Banach Lie triple system with norm $\|\cdot\|_{B}$.

Let $B^{A}$ be the set of all mappings from $A$ to $B$, let $\mathscr{L}(A, B)$ be the set of all $\mathbb{C}$-linear mappings from $A$ to $B$, let $\mathscr{H}(A, B)$ be the set of all Lie triple homomorphisms from $A$ to $B$, let $\mathscr{D}(A, A)$ be the set of all Lie triple derivations on $A$, and let $\mathbb{R}^{+}$be the set of all nonnegative reals.

\section{Stability of Homomorphisms on Normed Lie Triple Systems}

In the section, we prove the stability of homomorphisms on normed Lie triple systems associated with the Cauchy-Jensen additive equation. For $f \in B^{A}$, we define an operator $J_{\lambda} f$ : $A^{p+d} \rightarrow B$ by

$$
\begin{aligned}
J_{\lambda} f\left(x_{1}, \ldots, x_{p}, y_{1}, \ldots, y_{d}\right) \\
:=r_{0} f\left(\frac{s \sum_{j=1}^{p} \lambda x_{j}+t \sum_{j=1}^{d} \lambda y_{j}}{r_{0}}\right) \\
\quad-s \sum_{j=1}^{p} \lambda f\left(x_{j}\right)-t \sum_{j=1}^{d} \lambda f\left(y_{j}\right)
\end{aligned}
$$

for all $\lambda \in S$ and all $x_{1}, \ldots, x_{p}, y_{1}, \ldots, y_{d} \in A$, where $S$ is a nontrivial connected subset of $\mathbb{T}=\{z \in \mathbb{C}:|z|=1\}$ with $1 \in S$ and $S \backslash\{1\} \neq \emptyset$.

Lemma 1. Let $f \in B^{A}$ with $f(0)=0$. Then

$$
J_{\lambda} f\left(x_{1}, \ldots, x_{p}, y_{1}, \ldots, y_{d}\right)=0
$$

for all $\lambda \in S$ and all $x_{1}, \ldots, x_{p}, y_{1}, \ldots, y_{d} \in A$ if and only if $f \in \mathscr{L}(A, B)$.

Proof. Let $J_{\lambda} f=0$ for all $\lambda \in S$. Then we note that $r_{0} f\left(\left(s / r_{0}\right) x\right)=s f(x)$ and $r_{0} f\left(\left(t / r_{0}\right) y\right)=t f(y)$ for all $x, y \in A$. Thus, $r_{0} f\left((\lambda s x+\lambda t y) / r_{0}\right)=s \lambda f(x)+t \lambda f(y)=$ $\lambda r_{0} f\left(s x / r_{0}\right)+\lambda r_{0} f\left(t y / r_{0}\right)$ for all $\lambda \in S$. So $f(\lambda x+\lambda y)=$ $\lambda f(x)+\lambda f(y)$ for all $\lambda \in S$, and then $f$ is an additive mapping and $f(\lambda x)=\lambda f(x)$ for all $\lambda \in S$ and all $x \in A$. Hence the assumptions of Lemma 1 in [31] are fulfilled, for instance, in view of the comments in the papers $[32,33]$, and so we can deduce the lemma. The converse is trivial.

We first consider stability theorem for approximate Lie triple homomorphisms of the functional equation $J_{\lambda} f=0$ with action of $\lambda \in S$.

Theorem 2. Suppose that $f \in B^{A}$ with $f(0)=0$ satisfies

$$
\begin{gathered}
\left\|J_{\lambda} f\left(x_{1}, \ldots, x_{p}, y_{1}, \ldots, y_{d}\right)\right\|_{B} \leq \varphi\left(x_{1}, \ldots, x_{p}, y_{1}, \ldots, y_{d}\right), \\
\|f([x, y, z])-[f(x), f(y), f(z)]\|_{B} \leq \psi(x, y, z)
\end{gathered}
$$

for all $\lambda \in S$ and all $x_{1}, \ldots, x_{p}, y_{1}, \ldots, y_{d}, x, y, z \in$ A. If $\varphi$ : $A^{p+d} \rightarrow \mathbb{R}^{+}$and $\psi: A^{3} \rightarrow \mathbb{R}^{+}$are functions such that

$$
\widetilde{\varphi}(x):=\sum_{n=0}^{\infty} \frac{1}{|\alpha|^{n}} \varphi(\overbrace{0, \ldots, 0}^{p}, \overbrace{\alpha^{n} x, \ldots, \alpha^{n} x}^{d})<\infty,
$$

$$
\left(\tilde{\varphi}(x):=\sum_{n=1}^{\infty}|\alpha|^{n} \varphi(\overbrace{0, \ldots, 0}^{p}, \overbrace{\frac{x}{\alpha^{n}}, \ldots, \frac{x}{\alpha^{n}}}^{d})<\infty \text {, resp. }\right),
$$

$$
\begin{gathered}
\lim _{n \rightarrow \infty} \frac{1}{|\alpha|^{n}} \varphi\left(\alpha^{n} x_{1}, \ldots, \alpha^{n} x_{p}, \alpha^{n} y_{1}, \ldots, \alpha^{n} y_{d}\right)=0, \\
\left(\lim _{n \rightarrow \infty}|\alpha|^{n} \varphi\left(\frac{x_{1}}{\alpha^{n}}, \ldots, \frac{x_{p}}{\alpha^{n}}, \frac{y_{1}}{\alpha^{n}}, \ldots, \frac{y_{d}}{\alpha^{n}}\right)=0 \text {, resp. }\right), \\
\lim _{n \rightarrow \infty} \frac{1}{|\alpha|^{3 n}} \psi\left(\alpha^{n} x, \alpha^{n} y, \alpha^{n} z\right)=0, \\
\left(\lim _{n \rightarrow \infty}|\alpha|^{3 n} \psi\left(\frac{x}{\alpha^{n}}, \frac{y}{\alpha^{n}}, \frac{z}{\alpha^{n}}\right)=0 \text {, resp. }\right)
\end{gathered}
$$


for all $x, y, z, x_{1}, \ldots, x_{p}, y_{1}, \ldots, y_{d} \in A$, where $\alpha=t d / r_{0}$, then there exists a unique $h \in \mathscr{H}(A, B)$ such that

$$
\|f(x)-h(x)\|_{B} \leq \frac{1}{|\alpha|\left|r_{0}\right|} \tilde{\varphi}(x)=\frac{1}{|t| d} \widetilde{\varphi}(x)
$$

for all $x \in A$.

Proof. Letting $\lambda=1, x_{1}=\cdots=x_{p}=0$, and $y_{1}=\cdots=y_{d}=$ $x$ in (4) and dividing by $|t| d$, we get

$$
\begin{aligned}
\left\|f(x)-\frac{f(\alpha x)}{\alpha}\right\|_{B} & \leq \frac{\varphi(0, \ldots, 0, x, \ldots, x)}{|t| d} \\
& =\frac{\varphi(0, \ldots, 0, x, \ldots, x)}{|\alpha|\left|r_{0}\right|}
\end{aligned}
$$

for all $x \in A$. Replacing $x$ by $\alpha^{n} x$ in (10) and dividing both sides of (10) by $|\alpha|^{n}$, we get

$$
\left\|\frac{f\left(\alpha^{n+1} x\right)}{\alpha^{n+1}}-\frac{f\left(\alpha^{n} x\right)}{\alpha^{n}}\right\|_{B} \leq \frac{\varphi\left(0, \ldots, 0, \alpha^{n} x, \ldots, \alpha^{n} x\right)}{|\alpha|^{n+1}\left|r_{0}\right|}
$$

for all $x \in A$ and all nonnegative integers $n$. Therefore, one can use induction to show that

$$
\left\|\frac{f\left(\alpha^{n} x\right)}{\alpha^{n}}-\frac{f\left(\alpha^{m} x\right)}{\alpha^{m}}\right\|_{B} \leq \sum_{k=m}^{n-1} \frac{\varphi\left(0, \ldots, 0, \alpha^{k} x, \ldots, \alpha^{k} x\right)}{|\alpha|^{k+1}\left|r_{0}\right|}
$$

for all nonnegative integers $n>m$ and all $x \in A$. It follows from the convergence of the series (6) that the sequence $\left\{f\left(\alpha^{n} x\right) / \alpha^{n}\right\}$ is a Cauchy sequence in $B$. Thus, we may define a mapping $h: A \rightarrow B$ by

$$
h(x):=\lim _{n \rightarrow \infty} \frac{f\left(\alpha^{n} x\right)}{\alpha^{n}}
$$

for all $x \in A$. Letting $m=0$ in (12) and passing $n$ to infinity, we lead to the approximation (9). It follows from (4) that

$$
\begin{gathered}
\left\|r_{0} h\left(\frac{s \sum_{j=1}^{p} \lambda x_{j}+t \sum_{j=1}^{d} \lambda y_{j}}{r_{0}}\right)-s \sum_{j=1}^{p} \lambda h\left(x_{j}\right)-t \sum_{j=1}^{d} \lambda h\left(y_{j}\right)\right\|_{B} \\
=\lim _{n \rightarrow \infty} \frac{1}{|\alpha|^{n}} \| r_{0} f\left(\alpha^{n}\left(\frac{s \sum_{j=1}^{p} \lambda x_{j}+t \sum_{j=1}^{d} \lambda y_{j}}{r_{0}}\right)\right) \\
-s \sum_{j=1}^{p} \lambda f\left(\alpha^{n} x_{j}\right)-t \sum_{j=1}^{d} \lambda f\left(\alpha^{n} y_{j}\right) \|_{B} \\
\leq \lim _{n \rightarrow \infty} \frac{1}{|\alpha|^{n}} \varphi\left(\alpha^{n} x_{1}, \ldots, \alpha^{n} x_{p}, \alpha^{n} y_{1}, \ldots, \alpha^{n} y_{d}\right)=0
\end{gathered}
$$

for all $\lambda \in S$ and $x_{1}, \ldots, x_{p}, y_{1}, \ldots, y_{d} \in A$. Hence

$$
r_{0} h\left(\frac{s \sum_{j=1}^{p} \lambda x_{j}+t \sum_{j=1}^{d} \lambda y_{j}}{r_{0}}\right)=s \sum_{j=1}^{p} \lambda h\left(x_{j}\right)+t \sum_{j=1}^{d} \lambda h\left(y_{j}\right)
$$

for all $\lambda \in S$ and $x_{1}, \ldots, x_{p}, y_{1}, \ldots, y_{d} \in A$. So, $h \in \mathscr{L}(A, B)$ by Lemma 1 .

It follows from (5) that

$$
\begin{aligned}
& \|h([x, y, z])-[h(x), h(y), h(z)]\|_{B} \\
& =\lim _{n \rightarrow \infty} \frac{1}{|\alpha|^{3 n}} \| f\left(\left[\alpha^{n} x, \alpha^{n} y, \alpha^{n} z\right]\right) \\
& \quad-\left[f\left(\alpha^{n} x\right), f\left(\alpha^{n} y\right), f\left(\alpha^{n} z\right)\right] \|_{B} \\
& \leq \lim _{n \rightarrow \infty} \frac{1}{|\alpha|^{3 n}} \psi\left(\alpha^{n} x, \alpha^{n} y, \alpha^{n} z\right)=0
\end{aligned}
$$

for all $x, y, z \in A$. So, $h \in \mathscr{H}(A, B)$.

To prove the uniqueness of such homomorphism $h$ subject to (9), we assume that there exists another Lie triple homomorphism $h^{\prime}: A \rightarrow B$ satisfying (9). Obviously, we have $h^{\prime}\left(\alpha^{n} x\right)=\alpha^{n} h^{\prime}(x)$ and $h\left(\alpha^{n} x\right)=\alpha^{n} h(x)$ for all $x \in A$. By the triangle inequality, (9), and above equality, we have

$$
\begin{aligned}
\left\|h(x)-h^{\prime}(x)\right\|_{B}= & |\alpha|^{-n}\left\|h\left(\alpha^{n} x\right)-h^{\prime}\left(\alpha^{n} x\right)\right\|_{B} \\
\leq & |\alpha|^{-n}\left\|h\left(\alpha^{n} x\right)-f\left(\alpha^{n} x\right)\right\|_{B} \\
& +|\alpha|^{-n}\left\|f\left(\alpha^{n} x\right)-h^{\prime}\left(\alpha^{n} x\right)\right\|_{B} \\
\leq & \frac{2 \widetilde{\varphi}\left(\alpha^{n} x\right)}{|t||d||\alpha|^{n}}
\end{aligned}
$$

for all $n \in \mathbb{N}$. By letting $n \rightarrow \infty$, we get $h(x)=h^{\prime}(x)$ for any $x \in A$. This completes the proof.

Corollary 3. Let $\theta$ be a positive real number, let $\left|t d / r_{0}\right| \neq 1$, and let $r$ be a real number such that either $0<r<1$ or $r>1$. Suppose that $f \in B^{A}$ with $f(0)=0$ satisfies

$$
\begin{gathered}
\left\|J_{\lambda} f\left(x_{1}, \ldots, x_{p}, y_{1}, \ldots, y_{d}\right)\right\|_{B} \leq \theta\left(\sum_{j=1}^{p}\left\|x_{j}\right\|_{A}^{r}+\sum_{j=1}^{d}\left\|y_{j}\right\|_{A}^{r}\right) \\
\|f([x, y, z])-[f(x), f(y), f(z)]\|_{B} \\
\leq \theta\left(\|x\|_{A}^{3 r}+\|y\|_{A}^{3 r}+\|z\|_{A}^{3 r}\right)
\end{gathered}
$$

for all $\lambda \in S$ and all $x, y, z, x_{1}, \ldots, x_{p}, y_{1}, \ldots, y_{d} \in A$. Then there exists a unique $h \in \mathscr{H}(A, B)$ such that 


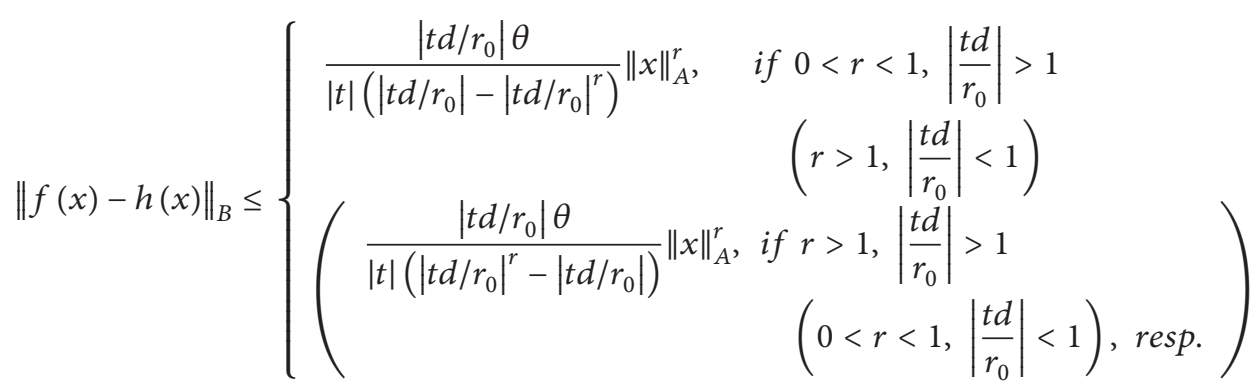

for all $x \in A$.

Proof. The proof follows from Theorem 2 by taking

$$
\begin{gathered}
\varphi\left(x_{1}, \ldots, x_{p}, y_{1}, \ldots, y_{d}\right):=\theta\left(\sum_{j=1}^{p}\left\|x_{j}\right\|_{A}^{r}+\sum_{j=1}^{d}\left\|y_{j}\right\|_{A}^{r}\right) \\
\psi(x, y, z):=\theta\left(\|x\|_{A}^{3 r}+\|y\|_{A}^{3 r}+\|z\|_{A}^{3 r}\right)
\end{gathered}
$$

for all $x, y, z, x_{1}, \ldots, x_{p}, y_{1}, \ldots, y_{d} \in A$.

Recently, the investigation on the hyperstability of the Cauchy and Jensen functional equations has been established $[34,35]$ and some information on it can be found in $[36,37]$. Now, we present the following hyperstability result associated with Lie triple homomorphisms between normed Lie triple systems.

From Theorems 2-5 in the paper [34], we can easily derive the following.

Theorem 4. Assume that $f: A \rightarrow B$ satisfies the inequality

$$
\begin{array}{r}
\left\|f\left(\frac{x+y}{2}\right)-\frac{f(x)+f(y)}{2}\right\|_{B} \leq \theta\|x\|_{A}^{p}\|y\|_{A}^{q}, \\
x, y \in A \backslash\{0\}, x+y \neq 0,
\end{array}
$$

for some $\theta \geq 0$, and $p, q \in \mathbb{R}, p+q \neq 0,1$. Then

$$
f\left(\frac{x+y}{2}\right)=\frac{f(x)+f(y)}{2}, \quad x, y \in A \backslash\{0\}, x+y \neq 0 .
$$

Corollary 5. Let $f \in B^{A}$ with $f(0)=0$. Assume that there exist $\theta \geq 0$ and $p, q \in \mathbb{R}$ with $p>0, p+q \neq 0,1$ such that

$$
\left\|2 f\left(\frac{\lambda x+\lambda y}{2}\right)-\lambda f(x)-\lambda f(y)\right\|_{B} \leq \theta\|x\|_{A}^{p}\|y\|_{A}^{q}
$$

for all $\lambda \in S$ and all $x, y \in A, y \neq 0$, and that a function $\psi:$ $A^{3} \rightarrow \mathbb{R}^{+}$satisfies the functional inequality (5) and (8) for all $x, y, z \in A$ and some $\alpha \in \mathbb{C} \backslash\{0\}$. Then $f \in \mathscr{H}(A, B)$.
Proof. By (23) with $\lambda=1$, condition (21) holds. Hence Theorem 4 implies that condition (22) is fulfilled. Next, with $x=0$ in (23), we get

$$
f\left(\lambda \frac{v}{2}\right)=\lambda \frac{f(v)}{2}, \quad v \in A, \lambda \in S
$$

which implies that

$$
f(2 v)=2 f(v), \quad f(\lambda v)=\lambda f(v), \quad v \in A, \lambda \in S .
$$

Hence, by (22),

$$
f(x+y)=f(x)+f(y), \quad x, y \in A \backslash\{0\}, x+y \neq 0,
$$

and next

$$
\begin{aligned}
f(u) & =f(2 u-u)=f(2 u)+f(-u) \\
& =2 f(u)+f(-u), \quad u \in A \backslash\{0\},
\end{aligned}
$$

which yields $f(-u)=-f(u)$ for $u \in A$. This and (26) show that $f$ is additive. Consequently, (26) and Lemma 1 in [31] jointly with the remarks in the papers $[32,33]$ imply the $\mathbb{C}$ linearity of $f$.

Further, it follows from (5) and (8) that, for all $x, y, z \in A$ and all $n \in \mathbb{N}$,

$$
\begin{aligned}
\| f( & (x, y, z])-[f(x), f(y), f(z)] \|_{B} \\
=\frac{1}{|\alpha|^{3 n}} \| f\left(\left[\alpha^{n} x, \alpha^{n} y, \alpha^{n} z\right]\right) & \quad-\left[f\left(\alpha^{n} x\right), f\left(\alpha^{n} y\right), f\left(\alpha^{n} z\right)\right] \|_{B} \\
& \leq \frac{1}{|\alpha|^{3 n}} \psi\left(\alpha^{n} x, \alpha^{n} y, \alpha^{n} z\right),
\end{aligned}
$$

which tends to zero as $n \rightarrow \infty$. So, $f \in \mathscr{H}(A, B)$.

Corollary 6. Let $f \in B^{A}$ with $f(0)=0$ and $a \in \mathbb{C} \backslash\{0\}$. Assume that there exist $\theta \geq 0$ and $p<0$ such that

$$
\|f(\lambda a x+\lambda a y)-\lambda a f(x)-\lambda a f(y)\|_{B} \leq \theta\left(\|x\|_{A}^{p}+\|y\|_{A}^{p}\right)
$$

for all $\lambda \in S$ and all $x, y \in A \backslash\{0\}$ and that a function $\psi$ : $A^{3} \rightarrow \mathbb{R}^{+}$satisfies the functional inequality (5) and (8) for all $x, y, z \in A$. Then $f \in \mathscr{H}(A, B)$. 
Proof. It follows from Theorem 2 in [38] (see also, Theorem 1.2 in [35]) and (29) that

$$
f(\lambda a x+\lambda a y)=\lambda a f(x)+\lambda a f(y)
$$

for all $x, y \in A \backslash\{0\}$ and all $\lambda \in S$. Thus,

$$
0=f(0)=f(a x-a x)=a f(x)+a f(-x), \quad x \in A \backslash\{0\},
$$

which implies $f(-x)=-f(x)$ for all $x \in A$. Now, using the equality (30), we get

$$
\begin{aligned}
f\left(a^{2} x\right) & =f\left(a^{2} x-a^{2} y+a^{2} y\right) \\
& =a f(a x-a y)+a f(a y) \\
& =a^{2} f(x)+a^{2} f(-y)+a f(a y) \\
& =a^{2} f(x)-a^{2} f(y)+a f(a y)
\end{aligned}
$$

for any $x, y \in A \backslash\{0\}$ with $x \neq y$. Thus, there is $c \in B$ defined as $c:=f\left(a^{2} x\right)-a^{2} f(x)=a f(a y)-a^{2} f(y)$ for any $x, y \in A \backslash\{0\}$, which yields $c / a=f\left(a^{2} x\right)-a f(a x), x \in A \backslash\{0\}$ and $c:=$ $f\left(a^{2} x\right)-a^{2} f(x)=f\left(a^{2} x\right)-a f(a x)+a f(a x)-a^{2} f(x)=c / a+c$ for any $x \in A \backslash\{0\}$. Consequently, $c=0$ and so $f(a x)=a f(x)$ for any $x \in A$. Therefore, we have

$$
f(a x+a y)=a f(x)+a f(y)=f(a x)+f(a y)
$$

for all $x, y \in A \backslash\{0\}$. Since $f(0)=0$, we conclude that $f$ is additive on $A$, and so we lead to the $\mathbb{C}$-linearity of $f$ by applying Lemma 1. The remaining proof is the same as the corresponding proof of Corollary 5.

Corollary 7. Let $f \in B^{A}$ with $f(0)=0$ and $a, b \in \mathbb{C} \backslash\{0\}$. Assume that there exist $\theta, p, q \in \mathbb{R}^{+}$with $p>0, p+q \neq 1$ such that

$$
\|f(\lambda a x+\lambda b y)-\lambda a f(x)-\lambda b f(y)\|_{B} \leq \theta\|x\|_{A}^{p}\|y\|_{A}^{q}
$$

for all $\lambda \in$ S and all $x, y \in A$ and that a function $\psi: A^{3} \rightarrow \mathbb{R}^{+}$ satisfies the functional inequality (5) and (8) for all $x, y, z \in A$. Then $f \in \mathscr{H}(A, B)$.

Proof. It follows from Theorem $20 \mathrm{in[36]}$ and (34) that

$$
f(\lambda a x+\lambda b y)=\lambda a f(x)+\lambda b f(y)
$$

for all $x, y \in A$ and all $\lambda \in S$. Thus, for all $x, y \in A$ and all $\lambda \in S$, one has $f(\lambda a x)=\lambda a f(x)$, which implies that $f$ is additive on $A$, and so we lead to the $\mathbb{C}$-linearity of $f$ by applying Lemma 1. The remaining proof is the same as the corresponding proof of Corollary 5.

Next, we consider stability theorem for approximate Lie triple homomorphisms of the functional equation $J_{\lambda} f=0$ with action of two scalars $\lambda=1$ and $\lambda=i \in \mathbb{C}$.
Theorem 8. Suppose that $f \in B^{A}$ with $f(0)=0$ satisfies

$$
\left\|J_{\lambda} f\left(x_{1}, \ldots, x_{p}, y_{1}, \ldots, y_{d}\right)\right\|_{B} \leq \varphi\left(x_{1}, \ldots, x_{p}, y_{1}, \ldots, y_{d}\right),
$$

$$
\|f([x, y, z])-[f(x), f(y), f(z)]\|_{B} \leq \psi(x, y, z)
$$

for $\lambda=1$ and $\lambda=i \in \mathbb{C}$ and for all $x_{1}, \ldots, x_{p}, y_{1}, \ldots, y$ ${ }_{d}, x, y, z \in A$. If $\varphi: A^{p+d} \rightarrow \mathbb{R}^{+}$and $\psi: A^{3} \rightarrow \mathbb{R}^{+}$are functions such that

$$
\begin{aligned}
& \widetilde{\varphi}(x):=\sum_{n=1}^{\infty}|\alpha|^{n} \varphi(\overbrace{0, \ldots, 0}^{p}, \frac{\overbrace{\frac{x}{\alpha^{n}}, \ldots, \frac{x}{\alpha^{n}}}^{d}}{d}<\infty, \\
& \left(\tilde{\varphi}(x):=\sum_{n=0}^{\infty} \frac{1}{|\alpha|^{n}} \varphi(\overbrace{0, \ldots, 0}^{p}, \overbrace{\alpha^{n} x, \ldots, \alpha^{n} x}^{d})<\infty, \text { resp. }\right), \\
& \lim _{n \rightarrow \infty}|\alpha|^{n} \varphi\left(\frac{x_{1}}{\alpha^{n}}, \ldots, \frac{x_{p}}{\alpha^{n}}, \frac{y_{1}}{\alpha^{n}}, \ldots, \frac{y_{d}}{\alpha^{n}}\right)=0, \\
& \left(\lim _{n \rightarrow \infty} \frac{1}{|\alpha|^{n}} \varphi\left(\alpha^{n} x_{1}, \ldots, \alpha^{n} x_{p}, \alpha^{n} y_{1}, \ldots, \alpha^{n} y_{d}\right)=0 \text {, resp. }\right) \text {, } \\
& \lim _{n \rightarrow \infty}|\alpha|^{3 n} \psi\left(\frac{x}{\alpha^{n}}, \frac{y}{\alpha^{n}}, \frac{z}{\alpha^{n}}\right)=0, \\
& \left(\lim _{n \rightarrow \infty} \frac{1}{|\alpha|^{3 n}} \psi\left(\alpha^{n} x, \alpha^{n} y, \alpha^{n} z\right)=0 \text {, resp. }\right)
\end{aligned}
$$

for all $x, y, z, x_{1}, \ldots, x_{p}, y_{1}, \ldots, y_{d} \in A$, where $\alpha=t d / r_{0}$, and the mapping $t \rightarrow f(t x)$ is continuous on $\mathbb{R}$ for each fixed $x \in$ $A$, then there exists a unique $h \in \mathscr{H}(A, B)$ such that

$$
\|f(x)-h(x)\|_{B} \leq \frac{1}{|\alpha|\left|r_{0}\right|} \tilde{\varphi}(x)=\frac{1}{|t| d} \widetilde{\varphi}(x)
$$

for all $x \in A$.

Proof. Applying the same argument as in the proof of Theorem 2, one can deduce the existence of a unique additive mapping $h: A \rightarrow B$ given by

$$
h(x):=\lim _{n \rightarrow \infty} \alpha^{n} f\left(\frac{x}{\alpha^{n}}\right)
$$

satisfying the required inequality (39). By the same reasoning as in the proof of the theorem of [4], the additive mapping $h$ is $\mathbb{R}$-linear.

Letting $\lambda=i, x_{1}=\cdots=x_{p}=0$, and $y_{1}=\cdots=y_{d}=x$ in (36), we have

$$
\left\|f(i x)-i \alpha f\left(\frac{x}{\alpha}\right)\right\|_{B} \leq \frac{\varphi(0, \ldots, 0, x / \alpha, \ldots, x / \alpha)}{\left|r_{0}\right|}
$$

for all $x \in A$. Then, it follows from the last inequality that

$$
\begin{aligned}
\left\|\alpha^{n} f\left(\frac{i x}{\alpha^{n}}\right)-i \alpha^{n+1} f\left(\frac{x}{\alpha^{n+1}}\right)\right\|_{B} \\
\leq \frac{|\alpha|^{n+1} \varphi\left(0, \ldots, 0, x / \alpha^{n+1}, \ldots, x / \alpha^{n+1}\right)}{|\alpha|\left|r_{0}\right|}
\end{aligned}
$$


for all $n \in \mathbb{N}$ and all $x \in A$. The right hand side tends to zero as $n \rightarrow \infty$, and so we obtain

$$
h(i x)=\lim _{n \rightarrow \infty} \alpha^{n} f\left(\frac{i x}{\alpha^{n}}\right)=\lim _{n \rightarrow \infty} i \alpha^{n+1} f\left(\frac{x}{\alpha^{n+1}}\right)=i h(x)
$$

for all $x \in A$. Therefore, for each $\mu=\lambda_{1}+i \lambda_{2} \in \mathbb{C}\left(\lambda_{1}, \lambda_{2} \in\right.$ $\mathbb{R})$,

$$
\begin{aligned}
h(\mu x)=h\left(\lambda_{1} x+i \lambda_{2} x\right) & =\lambda_{1} h(x)+\lambda_{2} h(i x) \\
& =\lambda_{1} h(x)+i \lambda_{2} h(x) \\
& =\left(\lambda_{1}+i \lambda_{2}\right) h(x) \\
& =\mu h(x)
\end{aligned}
$$

for all $x \in A$, so that $h \in \mathscr{L}(A, B)$. It follows from (37) that

$$
\begin{gathered}
\|h([x, y, z])-[h(x), h(y), h(z)]\|_{B} \\
\quad=\lim _{n \rightarrow \infty}|\alpha|^{3 n} \| f\left(\left[\frac{x}{\alpha^{n}}, \frac{y}{\alpha^{n}}, \frac{z}{\alpha^{n}}\right]\right)
\end{gathered}
$$

$$
\begin{array}{r}
-\left[f\left(\frac{x}{\alpha^{n}}\right), f\left(\frac{y}{\alpha^{n}}\right), f\left(\frac{z}{\alpha^{n}}\right)\right] \|_{B} \\
\leq \lim _{n \rightarrow \infty}|\alpha|^{3 n} \psi\left(\frac{x}{\alpha^{n}}, \frac{y}{\alpha^{n}}, \frac{z}{\alpha^{n}}\right)=0
\end{array}
$$

for all $x, y, z \in A$. So, $h \in \mathscr{H}(A, B)$.

The remainder of proof is similarly verified by the corresponding proof of Theorem 2.

Corollary 9. Let $\theta$ be a positive real number, let $\left|t d / r_{0}\right| \neq 1$, and let $r$ be a real number such that either $0<r<1$ or $r>1$. Suppose that $f \in B^{A}$ with $f(0)=0$ satisfies

$$
\begin{gathered}
\left\|J_{\lambda} f\left(x_{1}, \ldots, x_{p}, y_{1}, \ldots, y_{d}\right)\right\|_{B} \leq \theta\left(\sum_{j=1}^{p}\left\|x_{j}\right\|_{A}^{r}+\sum_{j=1}^{d}\left\|y_{j}\right\|_{A}^{r}\right), \\
\|f([x, y, z])-[f(x), f(y), f(z)]\|_{B} \\
\leq \theta\left(\|x\|_{A}^{3 r}+\|y\|_{A}^{3 r}+\|z\|_{A}^{3 r}\right)
\end{gathered}
$$

for all $\lambda=1$ and $\lambda=i \in \mathbb{C}$ and for all $x_{1}, \ldots, x_{p}, y_{1}, \ldots$, $y_{d}, x, y, z \in A$. If the mapping $t \rightarrow f(t x)$ is continuous on $\mathbb{R}$ for each fixed $x \in A$, then there exists a unique $h \in \mathscr{H}(A, B)$ such that

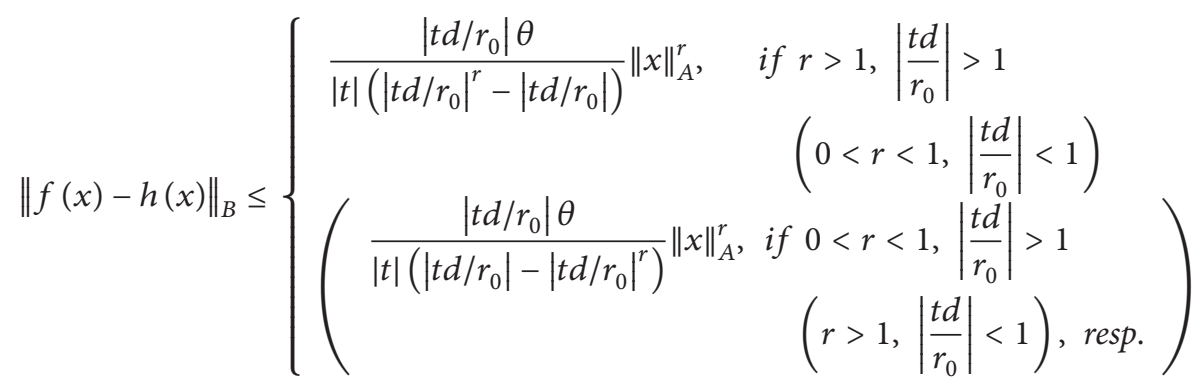

for all $x \in A$.

\section{Stability of Derivations on Normed Lie Triple Systems}

In this section, we prove the stability of derivations on normed Lie triple systems associated with the Cauchy-Jensen additive equation. Now, we consider stability theorem for approximate Lie triple derivations of the functional equation $J_{\lambda} f=0$ with action of $\lambda \in S$.

Theorem 10. Suppose that $f \in B^{B}$ with $f(0)=0$ satisfies

$$
\left\|J_{\lambda} f\left(x_{1}, \ldots, x_{p}, y_{1}, \ldots, y_{d}\right)\right\|_{B} \leq \varphi\left(x_{1}, \ldots, x_{p}, y_{1}, \ldots, y_{d}\right),
$$

$$
\begin{aligned}
& \|f([x, y, z])-[f(x), y, z]-[x, f(y), z]-[x, y, f(z)]\|_{B} \\
& \quad \leq \psi(x, y, z)
\end{aligned}
$$

for all $\lambda \in S$ and all $x_{1}, \ldots, x_{p}, y_{1}, \ldots, y_{d}, x, y, z \in B$. If $\varphi$ : $B^{p+d} \rightarrow \mathbb{R}^{+}$and $\psi: B^{3} \rightarrow \mathbb{R}^{+}$are functions such that

$$
\widetilde{\varphi}(x):=\sum_{n=0}^{\infty} \frac{1}{|\alpha|^{n}} \varphi\left(\alpha^{n} x, \ldots, \alpha^{n} x\right)<\infty,
$$$$
\left(\tilde{\varphi}(x):=\sum_{n=1}^{\infty}|\alpha|^{n} \varphi\left(\frac{x}{\alpha^{n}}, \ldots, \frac{x}{\alpha^{n}}\right)<\infty \text {, resp. }\right),
$$

$$
\begin{gathered}
\lim _{n \rightarrow \infty} \frac{1}{|\alpha|^{n}} \varphi\left(\alpha^{n} x_{1}, \ldots, \alpha^{n} x_{p}, \alpha^{n} y_{1}, \ldots, \alpha^{n} y_{d}\right)=0, \\
\left(\lim _{n \rightarrow \infty}|\alpha|^{n} \varphi\left(\frac{x_{1}}{\alpha^{n}}, \ldots, \frac{x_{p}}{\alpha^{n}}, \frac{y_{1}}{\alpha^{n}}, \ldots, \frac{y_{d}}{\alpha^{n}}\right)=0 \text {, resp. }\right),
\end{gathered}
$$




$$
\begin{gathered}
\lim _{n \rightarrow \infty} \frac{1}{|\alpha|^{3 n}} \psi\left(\alpha^{n} x, \alpha^{n} y, \alpha^{n} z\right)=0, \\
\left(\lim _{n \rightarrow \infty}|\alpha|^{3 n} \psi\left(\frac{x}{\alpha^{n}}, \frac{y}{\alpha^{n}}, \frac{z}{\alpha^{n}}\right)=0, \text { resp. }\right)
\end{gathered}
$$

for all $x, y, z, x_{1}, \ldots, x_{p}, y_{1}, \ldots, y_{d} \in B$, where $\alpha=(s p+t d) / r_{0}$, then there exists a unique $D \in \mathscr{D}(B, B)$, defined as $D(x)=$ $\lim _{n \rightarrow \infty}\left(f\left(\alpha^{n} x\right) / \alpha^{n}\right)\left(D(x)=\lim _{n \rightarrow \infty} \alpha^{n} f\left(x / \alpha^{n}\right)\right.$, resp. $)$, such that

$$
\|f(x)-D(x)\|_{B} \leq \frac{1}{|\alpha|\left|r_{0}\right|} \widetilde{\varphi}(x)=\frac{1}{|s p+t d|} \widetilde{\varphi}(x)
$$

for all $x \in B$.

Proof. Putting $\lambda=1$ and $x_{1}=\cdots=x_{p}=y_{1}, \cdots=y_{d}=x$ in (48), we obtain

$$
\left\|r_{0} f\left(\frac{s p+t d}{r_{0}} x\right)-(s p+t d) f(x)\right\|_{b} \leq \varphi(x, \ldots, x)
$$

for all $x \in B$. Let $\alpha:=(s p+t d) / r_{0}$; therefore

$$
\left\|f(x)-\frac{f(\alpha x)}{\alpha}\right\|_{B} \leq \frac{\varphi(x, \ldots, x)}{|\alpha|\left|r_{0}\right|}=\frac{1}{|s p+t d|} \varphi(x, \ldots, x)
$$

for all $x \in B$. Replacing $x$ by $\alpha^{n} x$ in (55) and dividing both sides of (55) by $|\alpha|^{n}$, we get

$$
\left\|\frac{f\left(\alpha^{n+1} x\right)}{\alpha^{n+1}}-\frac{f\left(\alpha^{n} x\right)}{\alpha^{n}}\right\|_{B} \leq \frac{\varphi\left(\alpha^{n} x, \ldots, \alpha^{n} x\right)}{|\alpha|^{n+1}\left|r_{0}\right|}
$$

for all $x \in B$ and all nonnegative integers $n$. Thus, one has

$$
\left\|\frac{f\left(\alpha^{n} x\right)}{\alpha^{n}}-\frac{f\left(\alpha^{m} x\right)}{\alpha^{m}}\right\|_{B} \leq \sum_{k=m}^{n-1} \frac{\varphi\left(\alpha^{k} x, \ldots, \alpha^{k} x\right)}{|\alpha|^{k+1}\left|r_{0}\right|}
$$

for all nonnegative integers $n>m$ and $x \in B$. It follows from the convergence of the series (50) that the sequence $\left\{f\left(\alpha^{n} x\right) / \alpha^{n}\right\}$ is a Cauchy sequence. From the completeness of $B$, this sequence converges in $B$. So we can define a mapping $D: B \rightarrow B$ by $D(x):=\lim _{n \rightarrow \infty}\left(f\left(\alpha^{n} x\right) / \alpha^{n}\right)$ for all $x \in B$.

Now, by considering $m=0$ and taking the limit as $n \rightarrow$ $\infty$ in (57), we obtain the estimation (53). It follows from (49) that

$$
\begin{aligned}
\| D([x, y, z])- & {[D(x), y, z]-[x, D(y), z]-[x, y, D(z)] \|_{B} } \\
=\lim _{n \rightarrow \infty} \frac{1}{|\alpha|^{3 n}} \| & f\left(\alpha^{3 n}[x, y, z]\right)-\left[f\left(\alpha^{n} x\right), \alpha^{n} y, \alpha^{n} z\right] \\
- & {\left[\alpha^{n} x, f\left(\alpha^{n} y\right), \alpha^{n} z\right] } \\
- & {\left[\alpha^{n} x, \alpha^{n} y, f\left(\alpha^{n} z\right)\right] \|_{B} } \\
\leq \lim _{n \rightarrow \infty} \frac{1}{|\alpha|^{3 n}} \psi & \left(\alpha^{n} x, \alpha^{n} y, \alpha^{n} z\right)=0
\end{aligned}
$$

for all $x, y, z \in B$. So, $D \in \mathscr{D}(B, B)$.

The remaining proof is similar to that of Theorem 2 .

Corollary 11. Assume that there exist real numbers $\theta \geq 0, \theta^{\prime} \geq$ 0 and that $r^{\prime}, s^{\prime}$, and $t^{\prime}$ are positive real numbers such that $r^{\prime}+$ $s^{\prime}+t^{\prime} \neq 3$. Let $\left|(s p+t d) / r_{0}\right| \neq 1$ and $r+s \neq 1$. Suppose $f \in B^{B}$ with $f(0)=0$ satisfies

$$
\begin{aligned}
& \left\|J_{\lambda} f\left(x_{1}, \ldots, x_{p}, y_{1}, \ldots, y_{d}\right)\right\|_{B} \leq \theta \prod_{i=1}^{p}\left\|x_{i}\right\|_{B}^{r_{i}} \prod_{j=1}^{d}\left\|y_{j}\right\|_{B}^{s_{j}}, \\
& \|f([x, y, z])-[f(x), y, z]-[x, f(y), z]-[x, y, f(z)]\|_{B} \\
& \leq \theta^{\prime}\|x\|_{B}^{r^{\prime}}\|y\|_{B}^{s^{\prime}}\|z\|_{B}^{t^{\prime}}
\end{aligned}
$$

for all $\lambda \in S$ and all $x_{1}, \ldots, x_{p}, y_{1}, \ldots, y_{d}, x, y, z \in B$. Then there exists a unique $D \in \mathscr{D}(B, B)$ such that

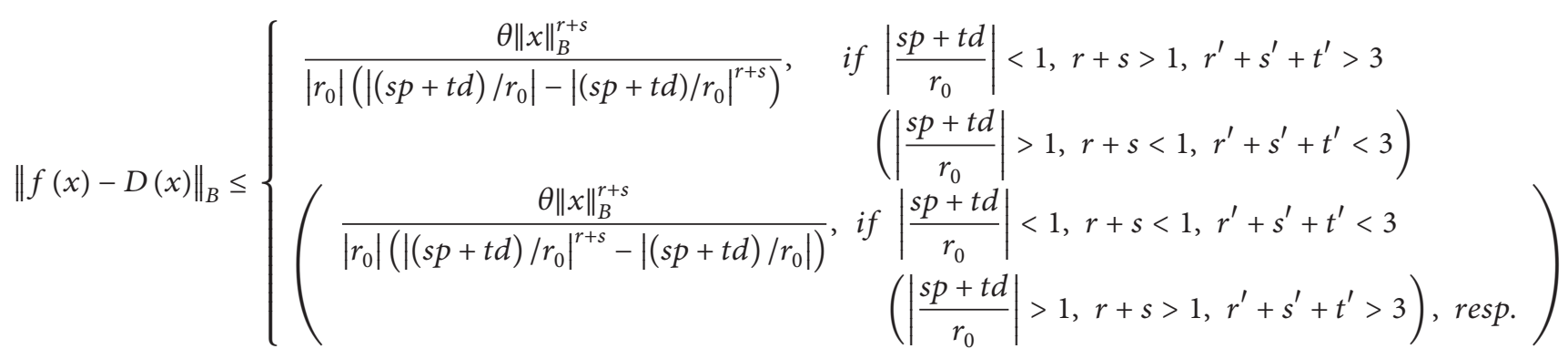

for all $x \in B$, where $r:=\sum_{i=1}^{p} r_{i}, s:=\sum_{j=1}^{d} s_{j}$.

Now, applying the above main theorem, we present the following hyperstability result associated with Lie triple derivations on Banach Lie triple systems.
Corollary 12. Let $f \in B^{B}$ with $f(0)=0$. Assume that there exist $\theta \geq 0$ and $p, q \in \mathbb{R}$ with $p>0, p+q \neq 0,1$ such that

$$
\left\|2 f\left(\frac{\lambda x+\lambda y}{2}\right)-\lambda f(x)-\lambda f(y)\right\|_{B} \leq \theta\|x\|_{A}^{p}\|y\|_{A}^{q}
$$


for all $\lambda \in S$ and all $x, y \in B, y \neq 0$ and that a function $\psi$ : $A^{3} \rightarrow \mathbb{R}^{+}$satisfies the functional inequality (49) and (52) for all $x, y, z \in A$ and some $\alpha \in \mathbb{C} \backslash\{0\}$. Then $f \in \mathscr{D}(B, B)$.

Proof. By the same reason as in the proof of Corollary 5, we lead to the $\mathbb{C}$-linearity of $f$. Further, it follows from (49) and (52) that for all $x, y, z \in B$ and all $n \in \mathbb{N}$

$$
\begin{gathered}
\|f([x, y, z])-[f(x), y, z]-[x, f(y), z]-[x, y, f(z)]\|_{B} \\
=\frac{1}{|\alpha|^{3 n}} \| f\left(\alpha^{3 n}[x, y, z]\right)-\left[f\left(\alpha^{n} x\right), \alpha^{n} y, \alpha^{n} z\right] \\
\quad-\left[\alpha^{n} x, f\left(\alpha^{n} y\right), \alpha^{n} z\right]-\left[\alpha^{n} x, \alpha^{n} y, f\left(\alpha^{n} z\right)\right] \|_{B} \\
\leq \frac{1}{|\alpha|^{3 n}} \psi\left(\alpha^{n} x, \alpha^{n} y, \alpha^{n} z\right)
\end{gathered}
$$

which tends to zero as $n \rightarrow \infty$. So, $f \in \mathscr{D}(B, B)$.

Corollary 13. Let $f \in B^{B}$ with $f(0)=0$ and $a \in \mathbb{C} \backslash\{0\}$. Assume that there exist $\theta \geq 0$ and $p<0$ such that

$$
\|f(\lambda a x+\lambda a y)-\lambda a f(x)-\lambda a f(y)\|_{B} \leq \theta\left(\|x\|_{B}^{p}+\|y\|_{B}^{p}\right)
$$

for all $\lambda \in S$ and all $x, y \in B \backslash\{0\}$ and that a function $\psi$ : $B^{3} \rightarrow \mathbb{R}^{+}$satisfies the functional inequality (49) and (52) for all $x, y, z \in A$. Then $f \in \mathscr{D}(B, B)$.

Corollary 14. Let $f \in B^{B}$ with $f(0)=0$ and $a, b \in \mathbb{C} \backslash\{0\}$. Assume that there exist $\theta, p, q \in \mathbb{R}^{+}$with $p>0, p+q \neq 1$ such that

$$
\|f(\lambda a x+\lambda b y)-\lambda a f(x)-\lambda b f(y)\|_{B} \leq \theta\|x\|_{A}^{p}\|y\|_{A}^{q}
$$

for all $\lambda \in S$ and all $x, y \in B$ and that a function $\psi: B^{3} \rightarrow \mathbb{R}^{+}$ satisfies the functional inequality (49) and (52) for all $x, y, z \in$ A. Then $f \in \mathscr{D}(B, B)$.

Next, we consider stability theorem for approximate Lie triple derivation of the functional equation $J_{\lambda} f=0$ with action of $\lambda=1$ and $\lambda=i \in \mathbb{C}$.

Theorem 15. Suppose that $f \in B^{B}$ with $f(0)=0$ satisfies

$$
\begin{aligned}
& \left\|J_{\lambda} f\left(x_{1}, \ldots, x_{p}, y_{1}, \ldots, y_{d}\right)\right\|_{B} \leq \varphi\left(x_{1}, \ldots, x_{p}, y_{1}, \ldots, y_{d}\right), \\
& \|f([x, y, z])-[f(x), y, z]-[x, f(y), z]-[x, y, f(z)]\|_{B} \\
& \quad \leq \psi(x, y, z)
\end{aligned}
$$

for $\lambda=1$ and $\lambda=i \in \mathbb{C}$ and for all $x_{1}, \ldots, x_{p}, y_{1}$, $\ldots, y_{d}, x, y, z \in B$. If $\varphi: B^{p+d} \rightarrow \mathbb{R}^{+}$and $\psi: B^{3} \rightarrow \mathbb{R}^{+}$ are functions such that

$$
\begin{aligned}
& \widetilde{\varphi}(x):=\sum_{n=1}^{\infty}|\alpha|^{n} \varphi\left(\frac{x}{\alpha^{n}}, \ldots, \frac{x}{\alpha^{n}}\right)<\infty, \\
& \left(\tilde{\varphi}(x):=\sum_{n=0}^{\infty} \frac{1}{|\alpha|^{n}} \varphi\left(\alpha^{n} x, \ldots, \alpha^{n} x\right)<\infty, \text { resp. }\right) \text {, } \\
& \lim _{n \rightarrow \infty}|\alpha|^{n} \varphi\left(\frac{x_{1}}{\alpha^{n}}, \ldots, \frac{x_{p}}{\alpha^{n}}, \frac{y_{1}}{\alpha^{n}}, \ldots, \frac{y_{d}}{\alpha^{n}}\right)=0, \\
& \left(\lim _{n \rightarrow \infty} \frac{1}{|\alpha|^{n}} \varphi\left(\alpha^{n} x_{1}, \ldots, \alpha^{n} x_{p}, \alpha^{n} y_{1}, \ldots, \alpha^{n} y_{d}\right)=0 \text {, resp. }\right) \text {, } \\
& \lim _{n \rightarrow \infty}|\alpha|^{3 n} \psi\left(\frac{x}{\alpha^{n}}, \frac{y}{\alpha^{n}}, \frac{z}{\alpha^{n}}\right)=0, \\
& \left(\lim _{n \rightarrow \infty} \frac{1}{|\alpha|^{3 n}} \psi\left(\alpha^{n} x, \alpha^{n} y, \alpha^{n} z\right)=0 \text {, resp. }\right)
\end{aligned}
$$

for all $x, y, z, x_{1}, \ldots, x_{p}, y_{1}, \ldots, y_{d} \in B$, where $\alpha=(s p+$ $t d) / r_{0}$, and the mapping $t \rightarrow f(t x)$ is continuous on $\mathbb{R}$ for each fixed $x \in B$, then there exists a unique $D \in$ $\mathscr{D}(B, B)$, defined as $D(x)=\lim _{n \rightarrow \infty} \alpha^{n} f\left(x / \alpha^{n}\right)(D(x)=$ $\lim _{n \rightarrow \infty}\left(f\left(\alpha^{n} x\right) / \alpha^{n}\right)$, resp. $)$, such that

$$
\|f(x)-D(x)\|_{B} \leq \frac{1}{|s p+t d|} \widetilde{\varphi}(x)
$$

for all $x \in B$.

Proof. The proof is similar to that of Theorem 10.

Corollary 16. Assume that there exist real numbers $\theta \geq 0$, $\theta^{\prime} \geq 0$ and that $r^{\prime}, s^{\prime}$, and $t^{\prime}$ are positive real numbers such that $r^{\prime}+s^{\prime}+t^{\prime} \neq 3$. Let $\left|(s p+t d) / r_{0}\right| \neq 1$ and $r+s \neq 1$. Suppose that $f \in B^{B}$ with $f(0)=0$ satisfies

$$
\begin{aligned}
& \left\|J_{\lambda} f\left(x_{1}, \ldots, x_{p}, y_{1}, \ldots, y_{d}\right)\right\|_{B} \leq \theta \prod_{i=1}^{p}\left\|x_{i}\right\|_{B}^{r_{i}} \prod_{j=1}^{d}\left\|y_{j}\right\|_{B}^{s_{j}}, \\
& \|f([x, y, z])-[f(x), y, z]-[x, f(y), z]-[x, y, f(z)]\|_{B} \\
& \leq \theta\|x\|_{B}^{r^{\prime}}\|y\|_{B}^{s^{\prime}}\|z\|_{B}^{t^{\prime}}
\end{aligned}
$$

for $\lambda=1$ and $\lambda=i \in \mathbb{C}$ and for all $x_{1}, \ldots, x_{p}$, $y_{1}, \ldots, y_{d}, x, y, z \in B$. If the mappingt $\rightarrow f(t x)$ is continuous on $\mathbb{R}$ for each fixed $x \in B$, then there exists a unique $D \in$ $\mathscr{D}(B, B)$ such that 


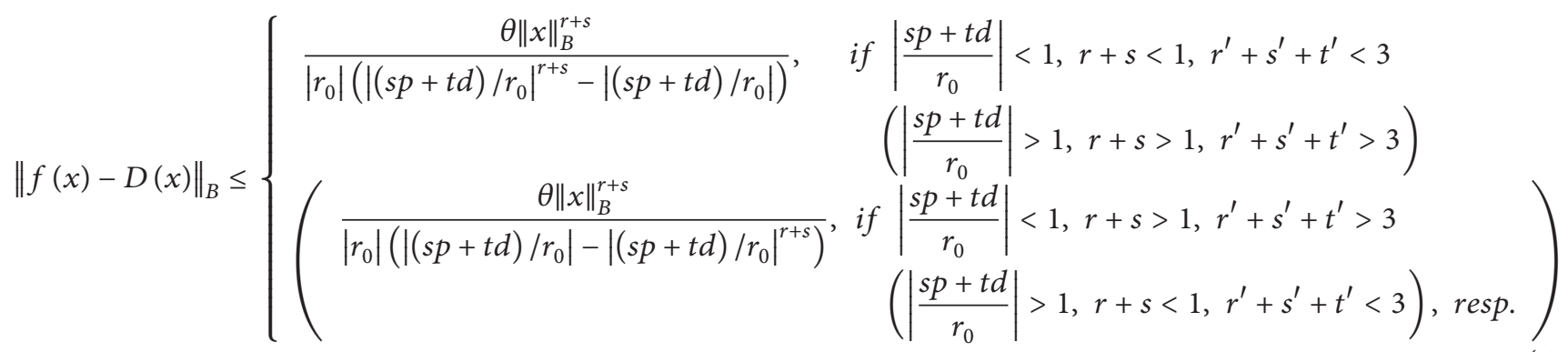

for all $x \in B$, where $r:=\sum_{i=1}^{p} r_{i}, s:=\sum_{j=1}^{d} s_{j}$.

\section{Conflict of Interests}

The authors declare that they have no competing interests.

\section{Authors' Contribution}

All authors carried out the proof. All authors conceived of the study and participated in its design and coordination. All authors read and approved the final version of the paper.

\section{Acknowledgments}

The authors would like to thank the reviewers and editors for their valuable comments and suggestions on the paper. This research was supported by Basic Science Research Program through the National Research Foundation of Korea (NRF) funded by the Ministry of Education (no. 2012R1A1A2008139).

\section{References}

[1] S. M. Ulam, Problems in Modern Mathematics, chapter VI, Science Editions, John Wiley \& Sons, New York, NY, USA, 1964.

[2] D. H. Hyers, "On the stability of the linear functional equation," Proceedings of the National Academy of Sciences of the United States of America, vol. 27, pp. 222-224, 1941.

[3] T. Aoki, "On the stability of the linear transformation in Banach spaces," Journal of the Mathematical Society of Japan, vol. 2, pp. 64-66, 1950.

[4] T. M. Rassias, "On the stability of the linear mapping in Banach spaces," Proceedings of the American Mathematical Society, vol. 72, no. 2, pp. 297-300, 1978.

[5] T. M. Rassias, "Problem 16; 2, report of the 27th international symposium on functional equations," Aequationes Mathematicae, vol. 39, pp. 292-293, 1990.

[6] Z. Gajda, "On stability of additive mappings," International Journal of Mathematics and Mathematical Sciences, vol. 14, no. 3, pp. 431-434, 1991.

[7] T. M. Rassias and P. Šemrl, "On the behavior of mappings which do not satisfy Hyers-Ulam stability," Proceedings of the American Mathematical Society, vol. 114, no. 4, pp. 989-993, 1992.
[8] N. Brillouët-Belluot, J. Brzdęk, and K. Ciepliński, "On some recent developments in Ulam's type stability," Abstract and Applied Analysis, vol. 2012, Article ID 716936, 41 pages, 2012.

[9] T. M. Rassias, "On the stability of functional equations in Banach spaces," Journal of Mathematical Analysis and Applications, vol. 251, no. 1, pp. 264-284, 2000.

[10] M. Bidkham, H. A. Soleiman Mezerji, and M. Eshaghi Gordji, "Hyers-Ulam stability of polynomial equations," Abstract and Applied Analysis, vol. 2010, Article ID 754120, 7 pages, 2010.

[11] X. Zhao, X. Yang, and C.-T. Pang, "Solution and stability of a general mixed type cubic and quartic functional equation," Journal of Function Spaces and Applications, vol. 2013, Article ID 673810, 8 pages, 2013.

[12] H.-M. Kim and J. Lee, "Approximate Euler-Lagrange quadratic mappings in fuzzy Banach spaces," Abstract and Applied Analysis, vol. 2013, Article ID 869274, 9 pages, 2013.

[13] A. Cayley, "On the 34 concomitants of the ternary cubic," American Journal of Mathematics, vol. 4, no. 1-4, pp. 1-15, 1881.

[14] M. Kapranov, I. M. Gelfand, and A. Zelevinsky, Discriminants, Results and Multidimensional Determinants, Birkhäuser, Basel, Switzerland, 1994.

[15] M. E. Gordji, A. Jabbari, and G. H. Kim, "Bounded approximate identities in ternary Banach algebras," Abstract and Applied Analysis, vol. 2012, Article ID 386785, 6 pages, 2012.

[16] P. Kannappan, Functional Equations and Inequalities with Applications, Springer Monographs in Mathematics, Springer, New York, NY, USA, 2009.

[17] M. Eshaghi Gordji, M. B. Ghaemi, J. M. Rassias, and B. Alizadeh, "Nearly ternary quadratic higher derivations on nonArchimedean ternary Banach algebras: a fixed point approach," Abstract and Applied Analysis, vol. 2011, Article ID 417187, 18 pages, 2011.

[18] G. L. Sewell, Quantum Mechanics and Its Emergent Macrophysics, Princeton University Press, Princeton, NJ, USA, 2002.

[19] M. Eshaghi Gordji, M. B. Ghaemi, S. Kaboli Gharetapeh, S. Shams, and A. Ebadian, "On the stability of $J^{*}$-derivations," Journal of Geometry and Physics, vol. 60, no. 3, pp. 454-459, 2010.

[20] M. Eshaghi Gordji and M. B. Savadkouhi, "Approximation of generalized homomorphisms in quasi-Banach algebras," Analele Stiintifice ale Universitatii Ovidius Constanta-Seria Matematica, vol. 17, no. 2, pp. 203-214, 2009. 
[21] M. Eshaghi Gordji and A. Najati, "Approximately $J^{*}$ homomorphisms: a fixed point approach," Journal of Geometry and Physics, vol. 60, no. 5, pp. 809-814, 2010.

[22] H. Zettl, "A characterization of ternary rings of operators," Advances in Mathematics, vol. 48, no. 2, pp. 117-143, 1983.

[23] W. G. Lister, "A structure theory of Lie triple systems," Transactions of the American Mathematical Society, vol. 72, pp. 217-242, 1952.

[24] N. C. Hopkins, "Nilpotent ideals in Lie and anti-Lie triple systems," Journal of Algebra, vol. 178, no. 2, pp. 480-492, 1995.

[25] J. Shokri, A. Ebadian, and R. Aghalary, "Hyers-Ulam-Rassias stability of homomorphisms and derivations on normed Lie triple systems," Thai Journal of Mathematics, vol. 9, no. 2, pp. 449-460, 2011.

[26] S. Helgason, Differential Geometry, Lie Group and Symmetric Spaces, vol. 80, American Mathematical Society, 1978.

[27] S. Okubo, Introduction to Octonion and Other Non-Associative Algebras in Physics, vol. 2 of Montroll Memorial Lecture Series in Mathematical Physics, Cambridge University Press, Cambridge, UK, 1995.

[28] N. Ghobadipour, A. Ebadian, T. M. Rassias, and M. Eshaghi Gordji, "A perturbation of double derivations on Banach algebras," Communications in Mathematical Analysis, vol. 11, no. 1, pp. 51-60, 2011.

[29] C.-G. Park, "Homomorphisms between Lie $J C^{*}$-algebras and Cauchy-Rassias stability of Lie JC* -algebra derivations," Journal of Lie Theory, vol. 15, no. 2, pp. 393-414, 2005.

[30] T. L. Shateri, "Superstability of generalized higher derivations," Abstract and Applied Analysis, vol. 2011, Article ID 239849, 9 pages, 2011.

[31] J. Brzdęk and A. Fošner, "Remarks on the stability of Lie homomorphisms," Journal of Mathematical Analysis and Applications, vol. 400, no. 2, pp. 585-596, 2013.

[32] W. Jabłoński, "On a class of sets connected with a convex function," Abhandlungen aus dem Mathematischen Seminar der Universität Hamburg, vol. 69, pp. 205-210, 1999.

[33] W. Jabłoński, "Sum of graphs of continuous functions and boundedness of additive operators," Journal of Mathematical Analysis and Applications, vol. 312, no. 2, pp. 527-534, 2005.

[34] A. Bahyrycz and M. Piszczek, "Hyperstability of the Jensen functional equation," Acta Mathematica Hungarica, vol. 142, no. 2, pp. 353-365, 2014.

[35] J. Brzdęk, "Hyperstability of the Cauchy equation on restricted domains," Acta Mathematica Hungarica, vol. 141, no. 1-2, pp. 5867, 2013 .

[36] J. Brzdęk and K. Ciepliński, "Hyperstability and superstability," Abstract and Applied Analysis, vol. 2013, Article ID 401756, 13 pages, 2013.

[37] G. Maksa and Z. Páles, "Hyperstability of a class of linear functional equations," Acta Mathematica. Academiae Paedagogicae Nyíregyháziensis, vol. 17, no. 2, pp. 107-112, 2001.

[38] M. Piszczek, "Remark on hyperstability of the general linear equation," Aequationes Mathematicae, 2013. 


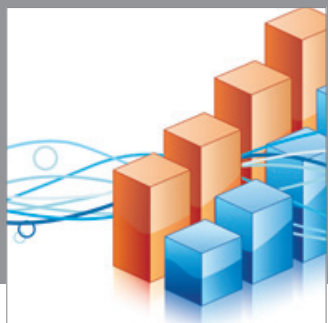

Advances in

Operations Research

mansans

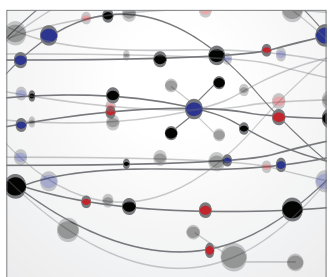

The Scientific World Journal
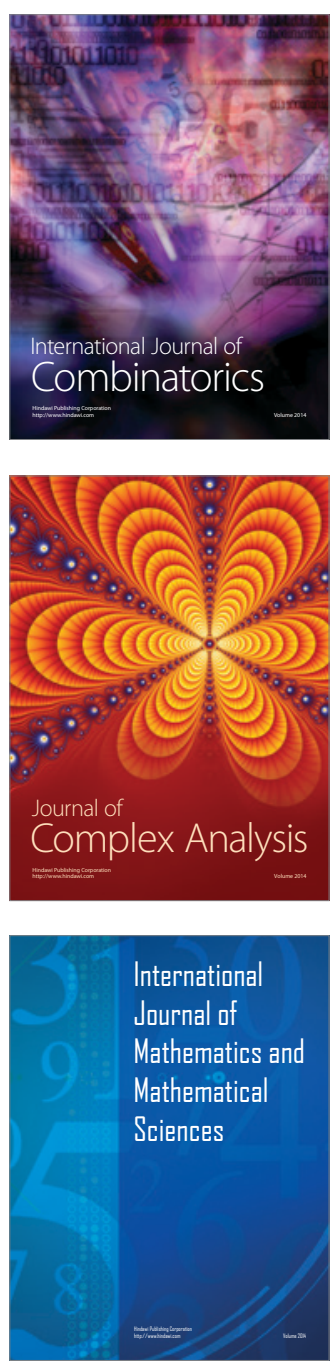
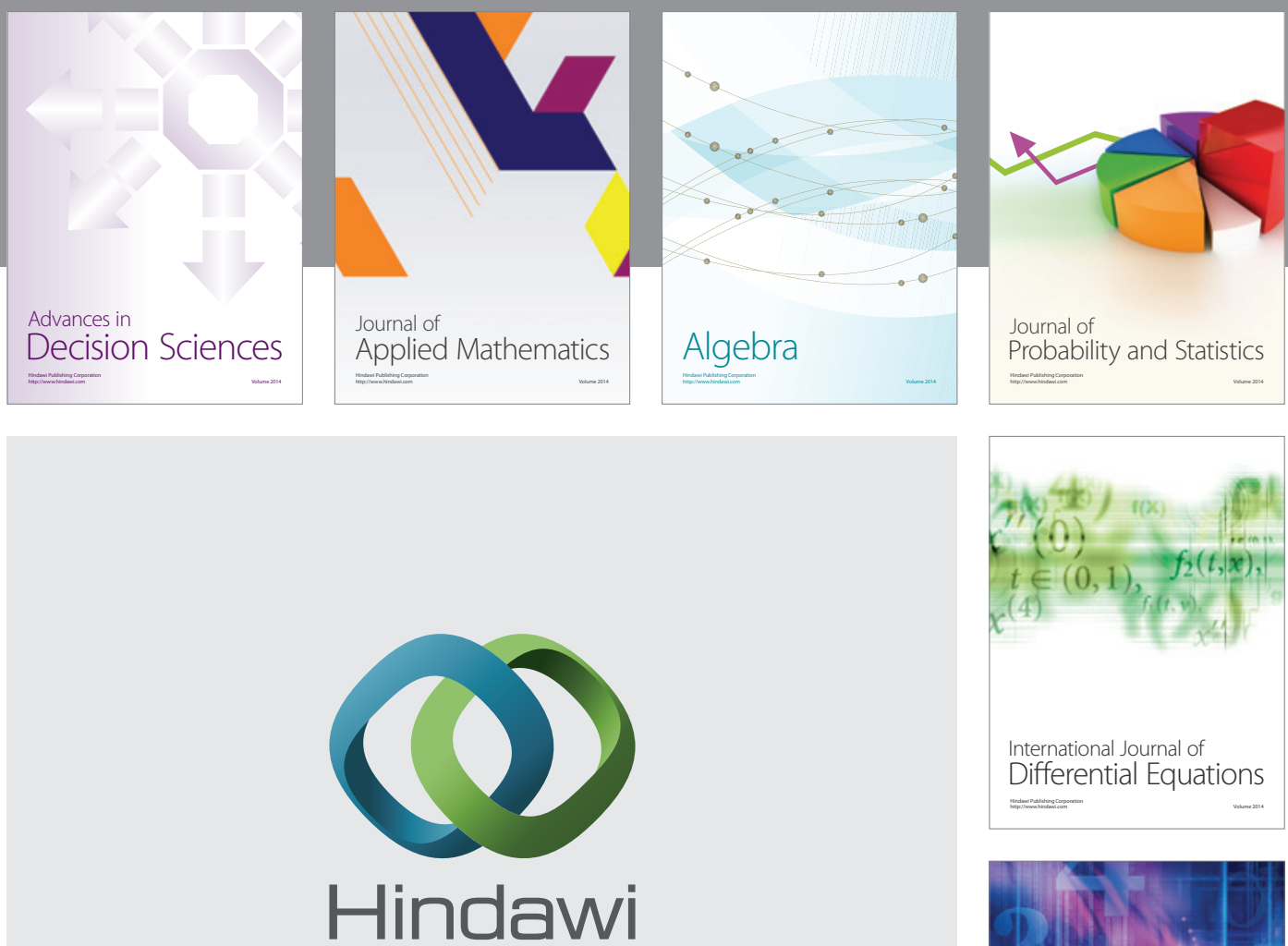

Submit your manuscripts at http://www.hindawi.com
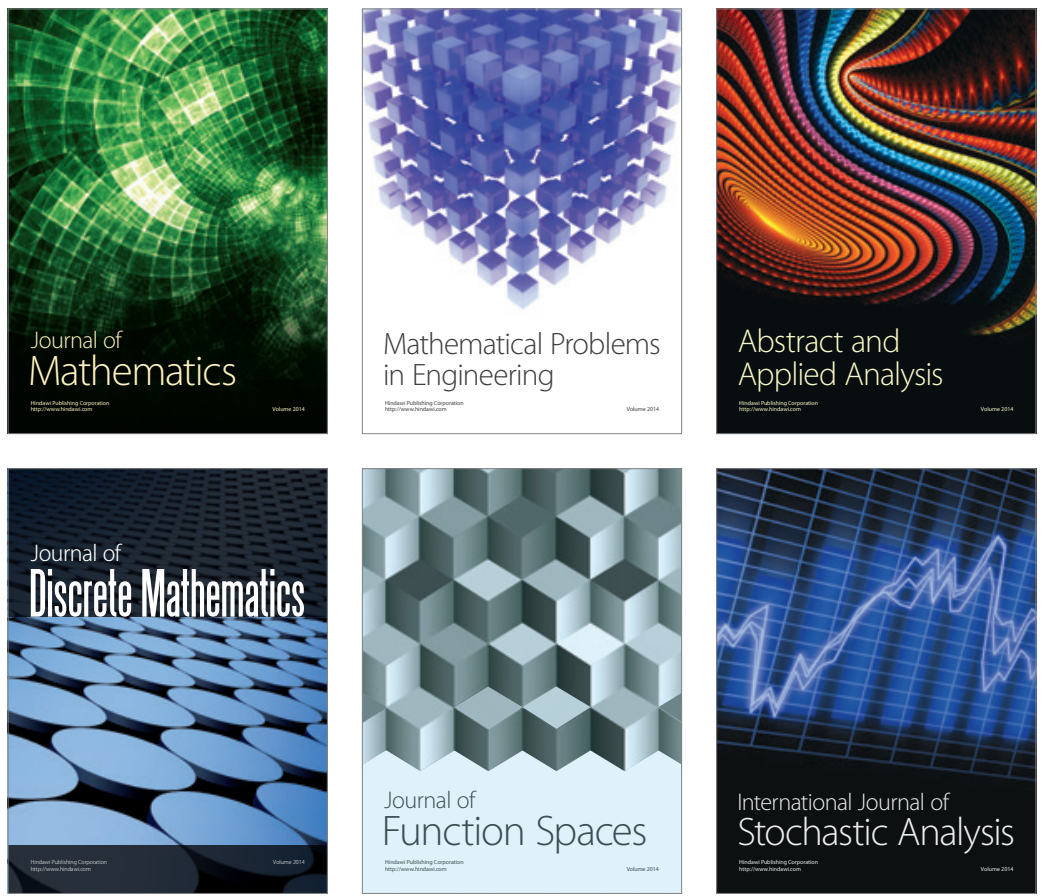

Journal of

Function Spaces

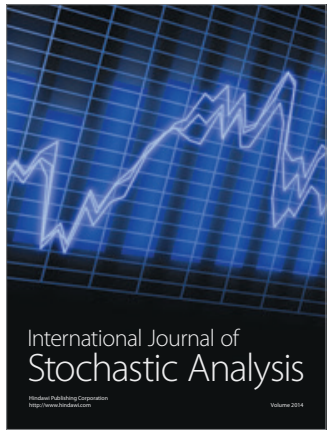

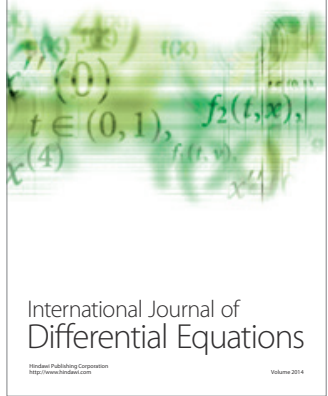
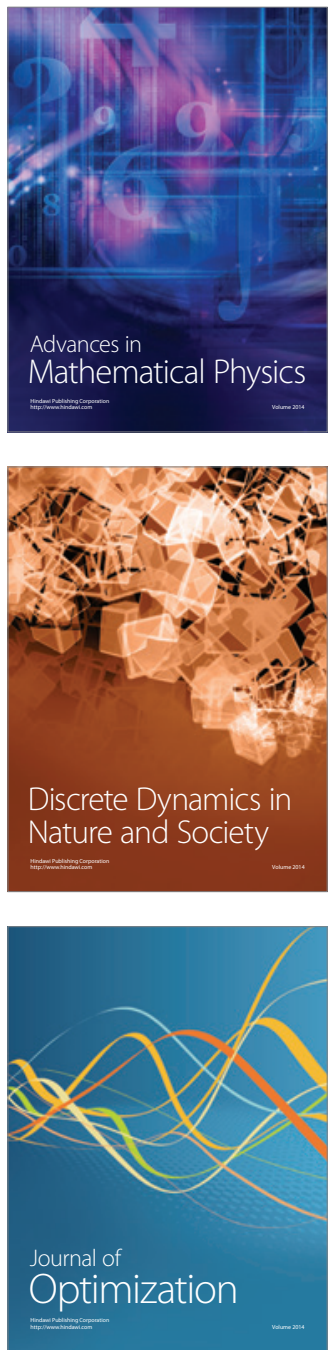